\title{
TIPOS DE ORQUIDÁCEAS BRENESIANAS, DESCRITAS POR R. SCHLECHTER, EN EL HERBARIO NACIONAL DE COSTA RICA
}

\author{
Silvia Lobo C. \\ Herbario Nacional de Costa Rica (CR), Museo Nacional de Costa Rica \\ Apdo. postal 749-1000 San José, Costa Rica, A.C. - museohn@racsa.co.cr
}

\begin{abstract}
This note informs about the presence of seven Brenesian orchid types, described by R. Schlechter, in the Herbario Nacional de Costa Rica (CR) type collection, whose existence was unknown.

RESUMEN. Esta nota revela la existencia, en el Herbario Nacional de Costa Rica (CR), de siete tipos de orquídeas brenesianas descritas por R. Schlechter, que no habían sido comunicados previamente.

Palabras clave / Key words: Type specimens, Orchidaceae, orchids, Herbario Nacional de Costa Rica (CR), R. Schlechter. A.M. Brenes.
\end{abstract}

La revisión de los tipos de las orquídeas de $\mathrm{R}$. Schlechter, realizada por K. Barringer (1986), incluye 89 de los 92 especímenes tipo recolectados por Alberto M. Brenes. Debido a la destrucción de muchos de los holotipos en el bombardeo de Berlín en 1943 y a lo complicado de la numeración de las muestras de A.M. Brenes (L.D. Gómez, citado por Barringer 1986), en ese momento no fue posible encontrar todos los ejemplares nombrados por Schlechter (1923); por esa razón, algunas de las tipificaciones fueron basadas en la colección de dibujos que se encuentra en el Oakes Ames Orchid Herbarium (AMES).

Durante la revisión y actualización de la colección de ejemplares tipo depositados en el Herbario Nacional de Costa Rica (CR), se hallaron siete ejemplares de orquídeas recolectados por A.M. Brenes, que corresponden a duplicados de los tipos incluidos en la lista de Barringer (1986), pero que no fueron mencionados como tipos por dicho autor. Esta nota tiene como fin informar a la comunidad científica la existencia en CR de dichos tipos, ya que algunos constituyen el único duplicado conocido hasta ahora. De cada ejemplar se indica el nombre original, la cita de la publicación original, la categoría del tipo asignada por Barringer (1986), el país, el nombre y el número de recolecta, el número de registro en $\mathrm{CR}$ y un apartado de observaciones sobre la condición general del ejemplar.
Epidendrum modestiflorum Schltr., Repert. Spec. Nov. Regni Veg. Beih. 19: 213. 1923. ISOLECTOTIPO: Costa Rica, A.M. Brenes 128 (CR 25822).

FIG. 1.

OBSERVACIONES: El espécimen es estéril; no obstante, la parte vegetativa está en buenas condiciones.

Epidendrum ramonianum Schltr., Repert. Spec. Nov. Regni Veg. Beih. 19: 217. 1923. ISOLECTOTIPO: Costa Rica, A.M. Brenes 101 (CR 25820).

FIG. 2.

OBSERVACIONES: El ejemplar es fértil y está en buenas condiciones.

Epidendrum serricardium Schltr., Repert. Spec. Nov. Regni Veg. Beih. 19: 218. 1923. ISOLECTOTIPO: Costa Rica, A.M. Brenes 254 (CR 33908).

FIG. 3.

OBSERVACIONES: El espécimen cuenta con pocas flores y su estado general es regular. Este nombre se considera sinónimo de E vincentinun Lindl. (Pupulin 2002).

Hexadesmia rigidipes Schltr., Repert. Spec. Nov. Regni Veg. Beih. 19: 206. 1923. ISOLECTOTIPO: Costa Rica, A.M. Brenes 145 (CR 25876). $\quad$ FIG. 4. Observaciones: El espécimen es fértil; no obstante, su condición es regular. Este nombre se considera sinónimo de Scaphyglottis lindeniana (A. Rich. \& Gal.) L. O. Williams (Pupulin 2002). 


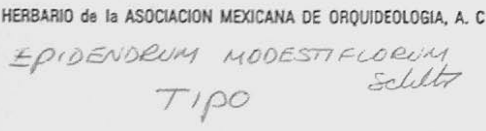

HERBARIO de la ASOCIACION MEXICANA DE ORQUIDEOLOGIA, A. C. EPRDENDRUA MODESTIFCORvin

$$
\text { Tipo }
$$

Determinó: Eric Hägsater Espe 1813 $19+3$ REC.
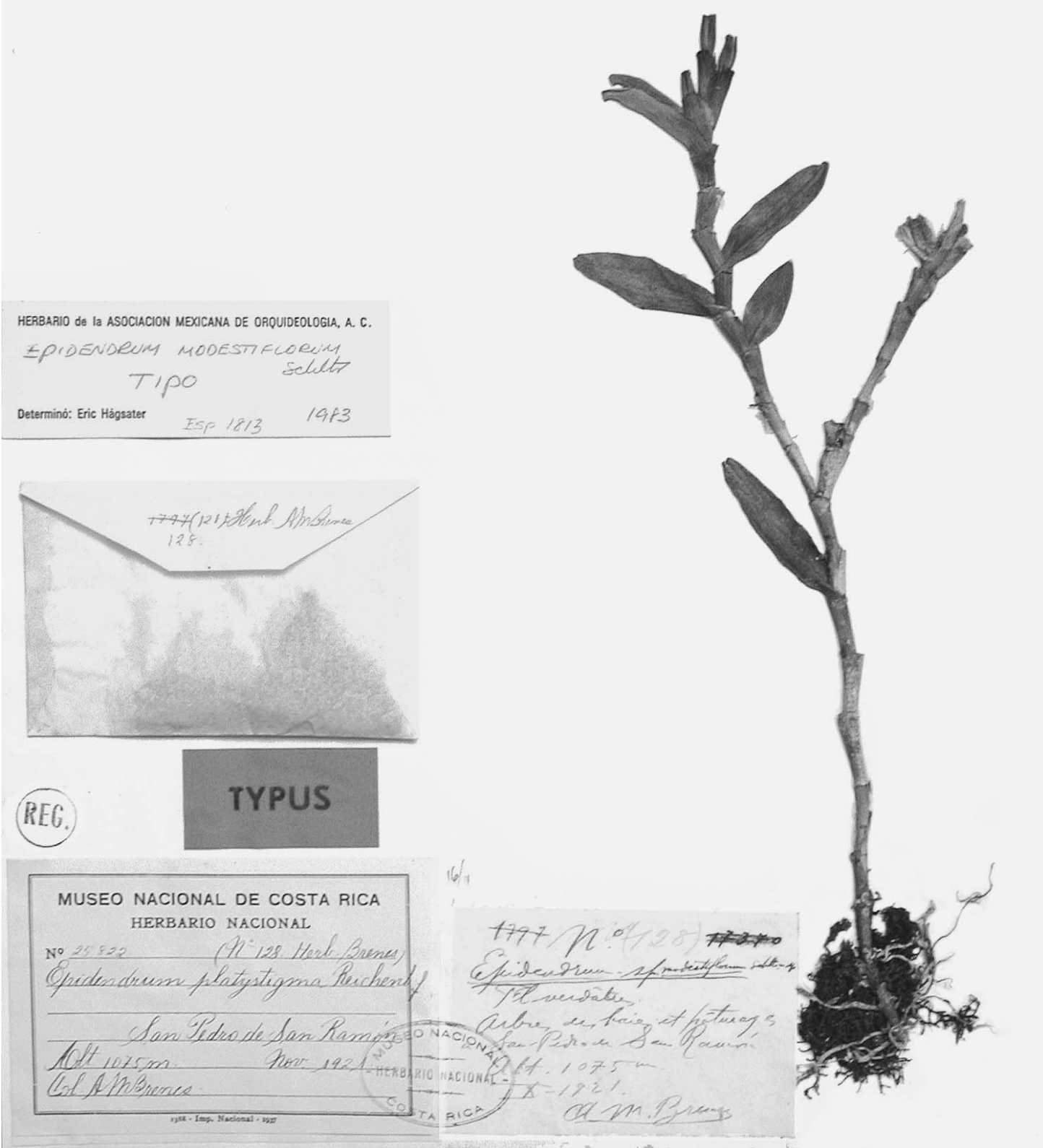

Figura 1: Isolectotipo de Epidendrum modestiflorum Schltr. 


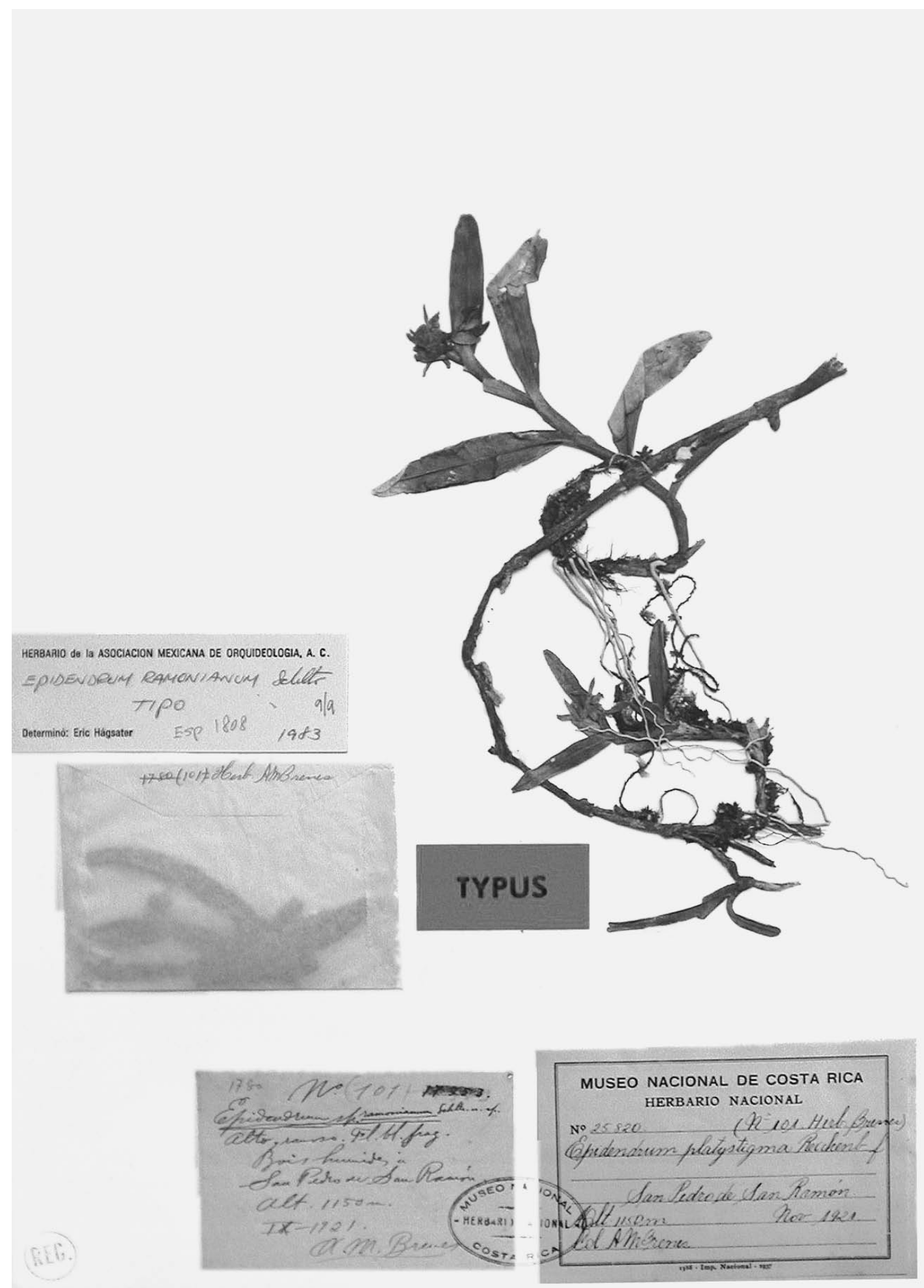

Figura 2: Isolectotipo de Epidendrum ramonianum Schltr. 


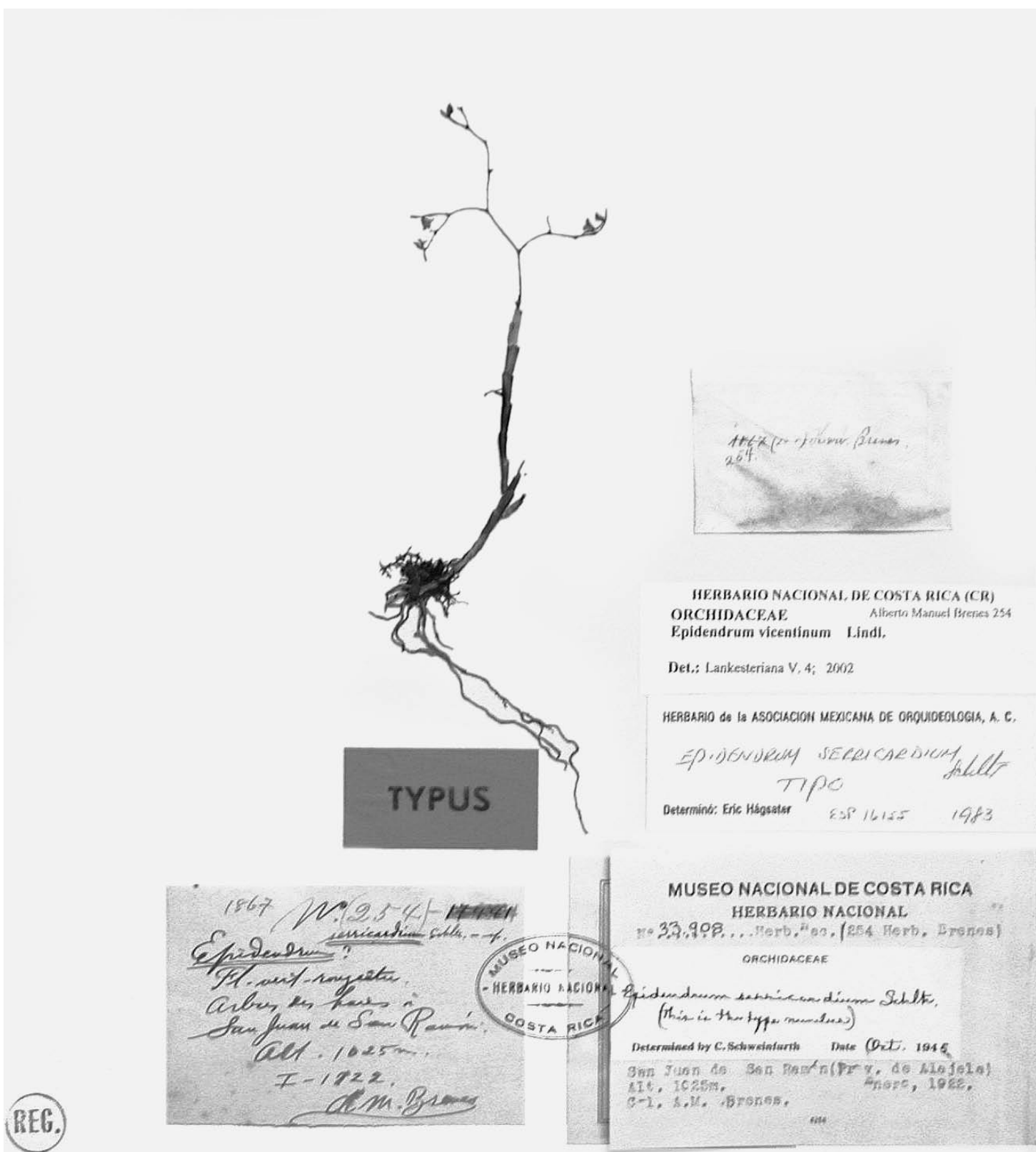

Figura 3: Isolectotipo de Epidendrum serricardium Schltr.

Maxillaria acutifolia Schltr., Repert. Spec. Nov. Regni Veg Beih. 19: 229. 1923. ISONEOTIPO: Costa Rica, A.M. Brenes 173(504) (CR 25964).

FIG. 5.

OBSERVACIONES: El ejemplar es fértil y se halla en buenas condiciones. Este nombre se considera sinónimo de M. angustissima Ames, F.T. Hubb. \& C. Schweinf. (Pupulin 2002).
Pleurothallis ramonensis Schltr., Repert. Spec. Nov. Regni Veg. Beih. 19: 193. 1923. ISOLECTOTIPO: Costa Rica, A.M. Brenes 88 (CR 26195). $\quad$ FIG. 6. OBSERVACIONES: Este ejemplar no se encuentra en buenas condiciones y posee una única flor que está separada de la planta. Este nombre se considera sinónimo de Stelis alajuelensis Pridgeon \& M. W. Chase (Pupulin 2002). 


\section{TIPO}
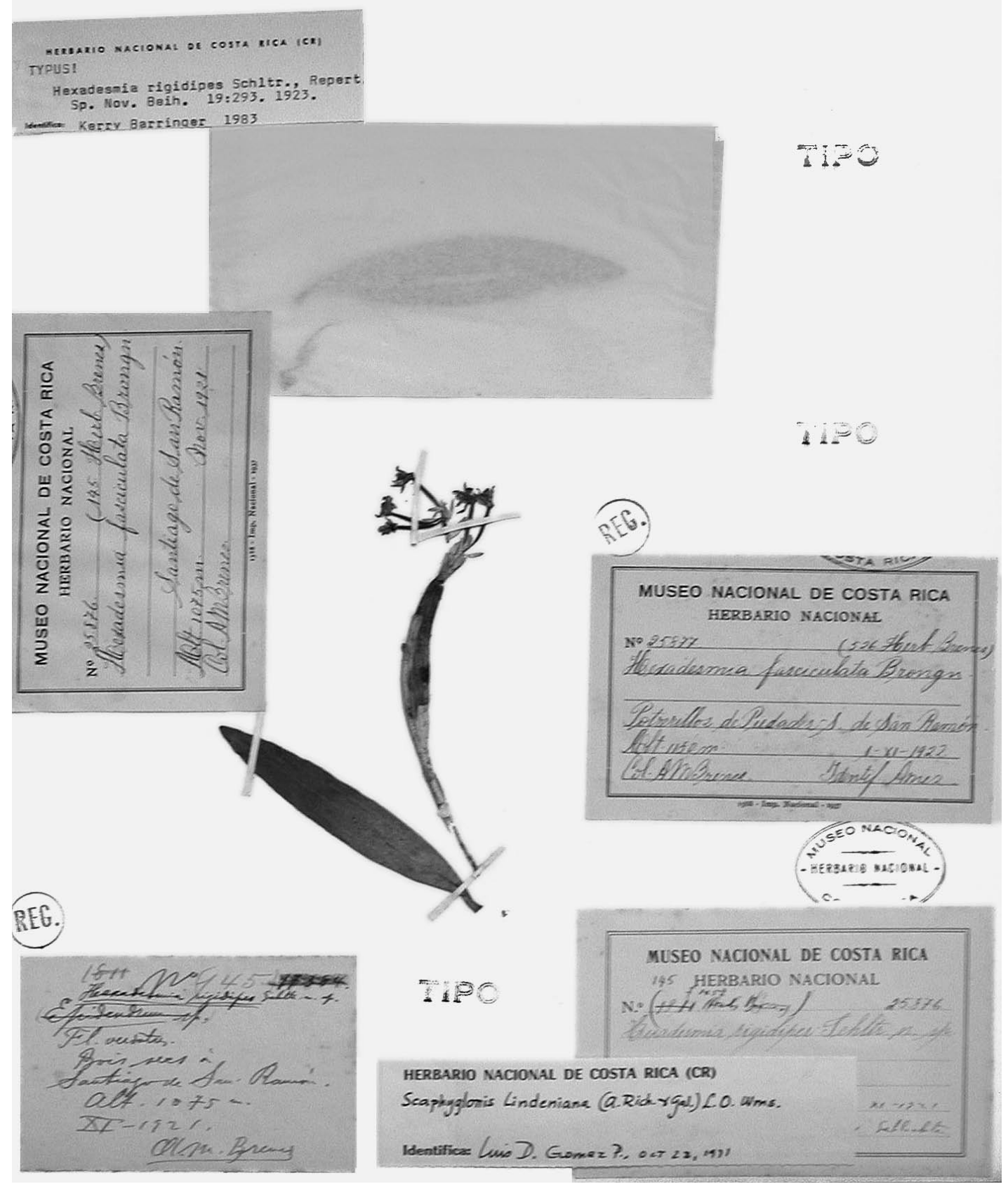

Figura 4: Isolectotipo de Hexadesmia rigidipes Schltr. 


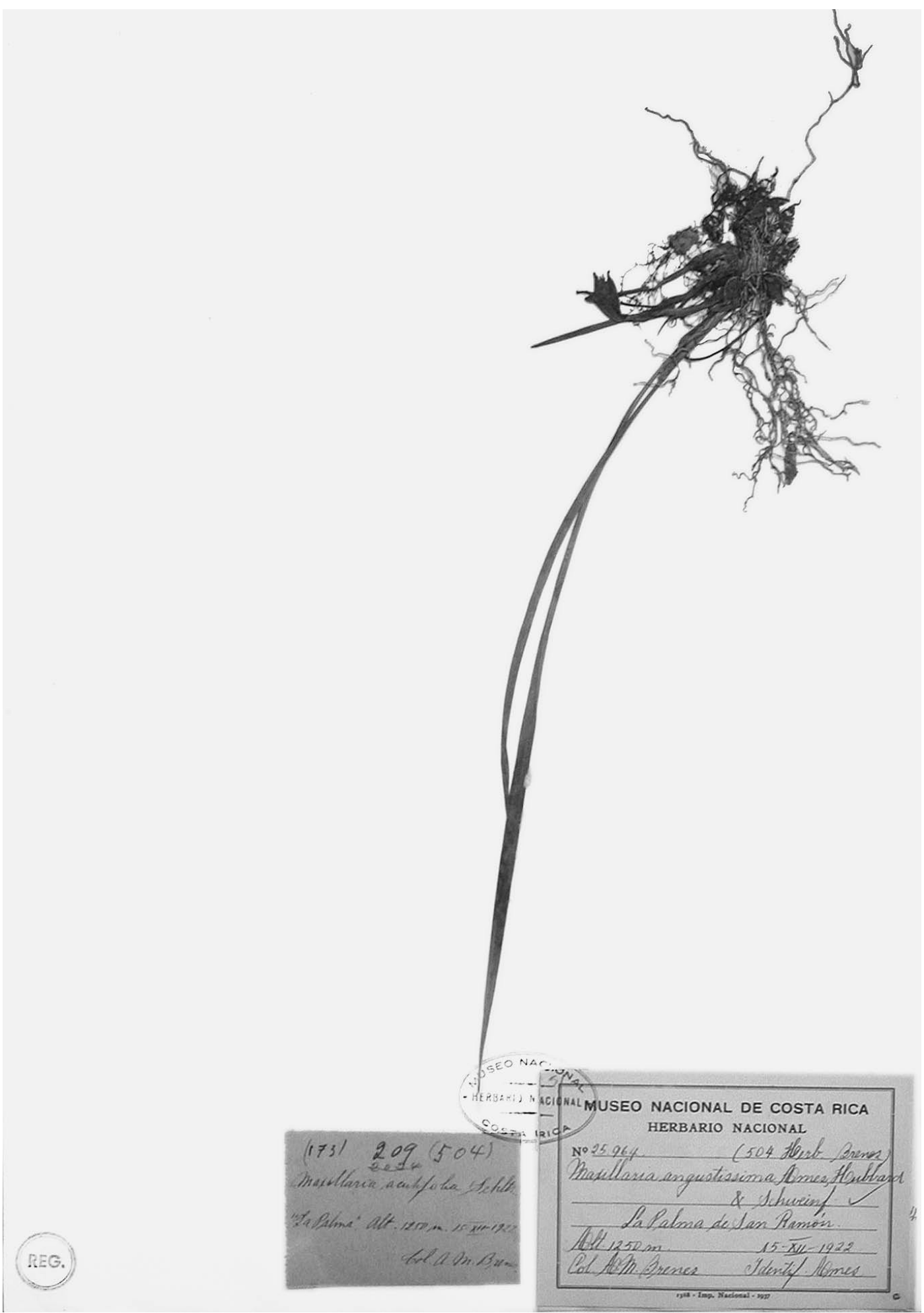

Figura 5: Isoneotipo de Maxillaria acutifolia Schltr. 


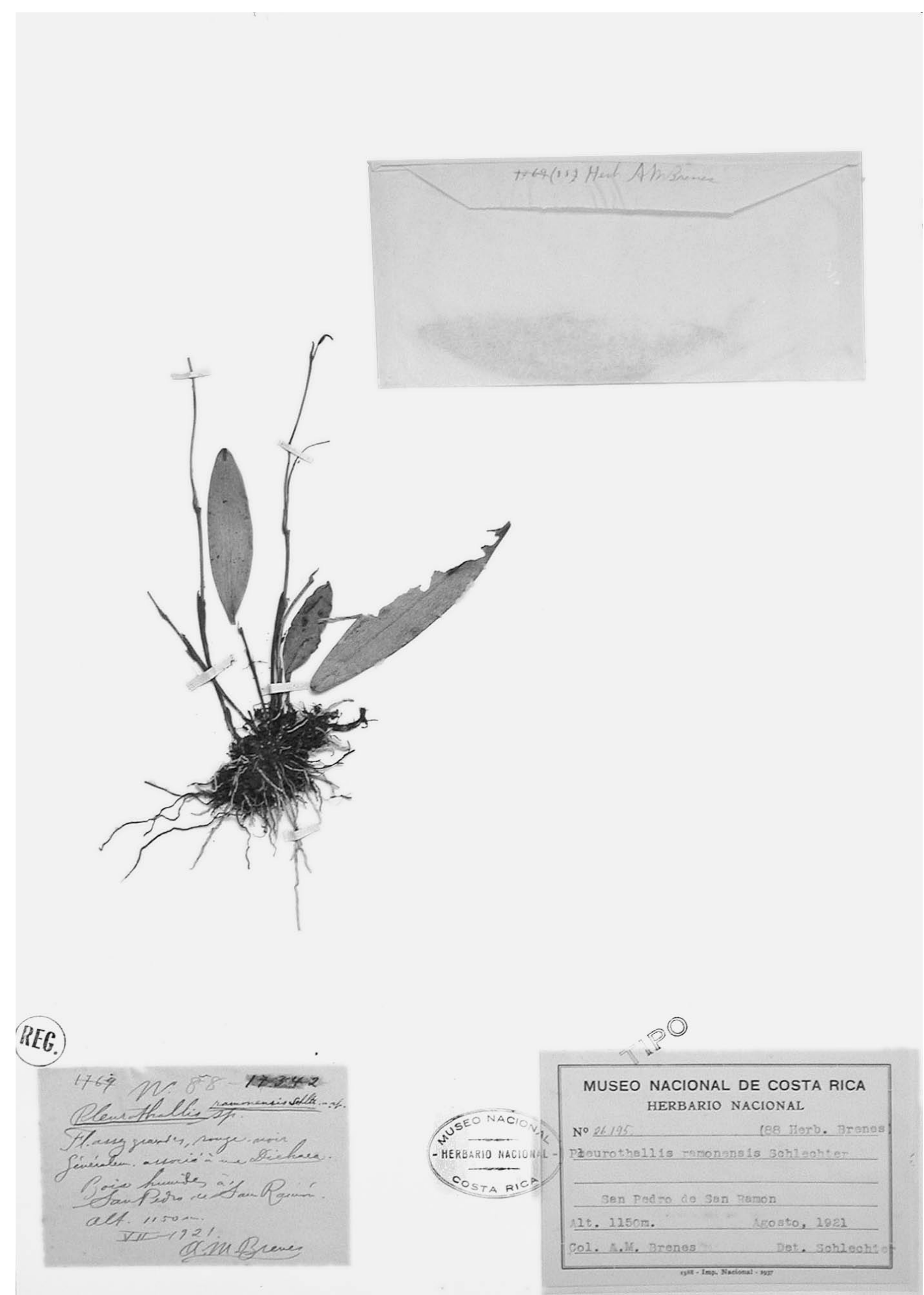

Figura 6: Isolectotipo de Pleurothallis ramonensis Schltr. 


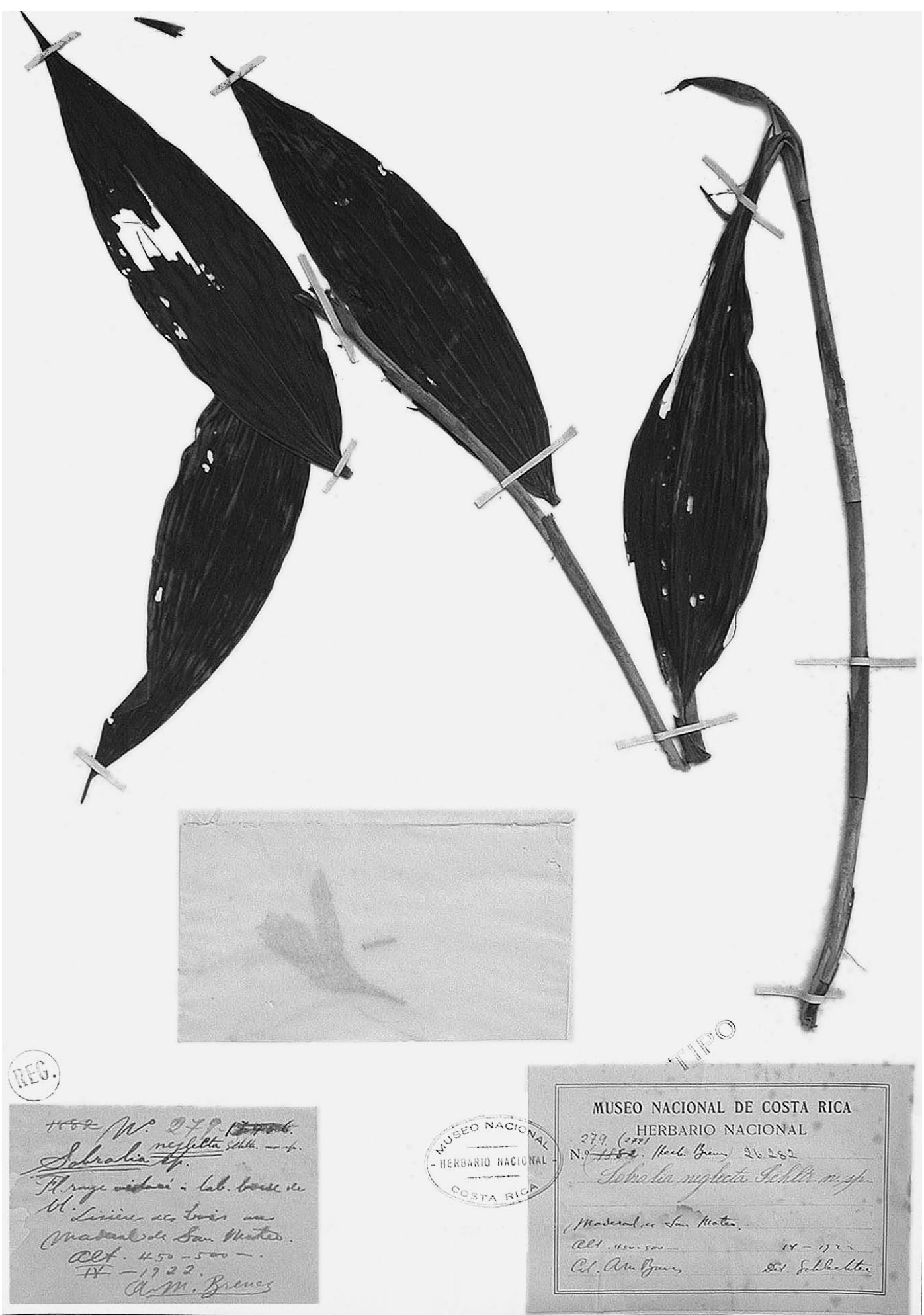

Figura 7: Isolectotipo de Sobralia neglecta Schltr. 
Sobralia neglecta Schltr., Repert. Spec. Nov. Regni Veg. Beih. 19: 161. 1923. ISOLECTOTIPO: Costa Rica, A.M. Brenes 279 (CR 26282).

FIG. 7.

OBSERVACIONES: El espécimen está en una condición regular y posee una única flor separada del resto del material.

Agradecimientos. Deseo agradecer al Dr. Carlos O. Morales (USJ) por sus valiosas observaciones y contribuciones a este manuscrito.

\section{LITERATURA CITADA}

Barringer, K. 1986. Typification of Schlechter's Costa Rican Orchidaceae. I. Types collected by A. Brenes. Fieldiana, Bot. n.s. 17: 1-24.

Pupulin, F. 2002. Catálogo revisado y anotado de las Orchidaceae de Costa Rica. Lankesteriana 4: 1-88.

Schlechter, R. 1923. Beiträge zur Orchideenkunde von Zentralamerika. II. Additamenta ad Orchideologiam Costaricensem. Orchidaceae Brenesianae. Repert. Spec. Nov. Regni Veg. Beih. 19: 158-269. 